\title{
Flow and heat transfer at a general three-dimensional stagnation point in a nanofluid.
}

\begin{abstract}
The problem dealing with steady three-dimensional stagnation point flow in a nanofluid is investigated. By using appropriate transformation for velocity and temperature, the basic equations governing the flow and heat transfer are reduced to a set of ordinary differential equations. These equations subjected to the associated boundary conditions are then solved numerically. The effects of the nanoparticle volume fraction $\varphi$ and the ratio of the gradient of velocities $\mathrm{c}$ on the flow and heat transfer characteristics are thoroughly examined.
\end{abstract}

Keyword: Nanofluids; Nodal point; Saddle point; Heat transfer; Numerical solution. 